\title{
Implant-based breast reconstruction: re-analysis of the odds ratio for skin flap necrosis
}

\author{
Andrea Messori $^{1}$ [D $\cdot$ Laura Bartoli $^{1} \cdot$ Elisa Ferracane $^{1}$
}

Received: 25 September 2020 / Accepted: 26 October 2020 / Published online: 22 November 2020

(c) Springer Science+Business Media, LLC, part of Springer Nature 2020

\section{Dear Editor,}

The paper by Abbate et al. [1] contains an error on page 5 ("significantly lower odds of mastectomy skin flap necrosis in the prepectoral group compared their subpectoral counterparts; odds ratio $0.02,95 \%$ CI $0.01-0.03, p<0.01$ ") because the correct odds ratio for the above mentioned comparison is the one reported in Table 9 ("skin necrosis: odds ratio, 0.665: $95 \%$ CI 0.464-0.952"). This more than 20-fold error is not completely casual because it results from an irrational design of Fig. 2. In fact, Fig. 2 describes 7 different variables presented according to 7 different logarithmic scales for the $\mathrm{x}$-axis, which of course are not comparable with one another. This creates some confusion in reading this paper, which is confirmed by the error made by the authors in reporting the odds ratio for skin flap necrosis.

\section{Reference}

1. Abbate O, Rosado N, Sobti N, Vieira BL, Liao EC (2020) Metaanalysis of prepectoral implant-based breast reconstruction: guide to patient selection and current outcomes. Breast Cancer Res Treat 182(3):543-554. https://doi.org/10.1007/s10549-020-05722-2

Publisher's Note Springer Nature remains neutral with regard to jurisdictional claims in published maps and institutional affiliations.
Andrea Messori

andrea.messori.it@gmail.com;

andrea.messori@regione.toscana.it

1 Regione Toscana, Via di San Salvi 12, 50135 Florence, Italy 